\title{
MXED MAGNETOCONVECTION IN A LID-DRIVEN CAVTY WTH A SINUSOIDAL WAVY WALL AND A CENTRAL HEAT CONDUCTING BODY
}

\section{Rehena Nasrin}

Department of Mathematics, Bangladesh University of Engineering and Technology, Dhaka-1000, Bangladesh.

Email: rehena@math.buet.ac.bd

\begin{abstract}
:
A numerical study has been performed to explore the mixed magnetoconvective flow and heat transfer characteristics of fluid contained in a lid-driven cavity having a sinusoidal wavy vertical surface. A heat conducting square body is located at the centre of cavity. The cavity horizontal walls are perfectly insulated while the corrugated right vertical surface is maintained at a uniform temperature higher than the left lid. The flow is assumed to be two-dimensional and Joule heating effect is considered. Calculations are carried out through solving governing equations for different parameters by using Galarkin's weighted residual finite element method. The flow pattern and the heat transfer characteristics inside the cavity are presented in the form of streamlines, isotherms, average temperature of the fluid and temperature of solid body centre for various values of Prandtl number Pr, Richardson number Ri and magnetic parameter Ha. The heat transfer rate is detected maximum for the highest Pr and absence of magnetic field.
\end{abstract}

Keywords: Mixed magnetoconvection, wavy wall, cavity, finite element method, heat conducting body.

\section{NOMENCLATURE}

\begin{tabular}{llll}
$B_{0}$ & magnetic field strength $\left(\mathrm{Wbm}^{-2}\right)$ & $T_{\text {hot }}$ & dimensional temperature of wavy wall (K) \\
$C_{p}$ & specific heat at constant pressure $\left(\mathrm{JKg}^{-1} \mathrm{~K}^{-1}\right)$ & $\Delta T$ & dimensional temperature difference $(\mathrm{K})$ \\
$d$ & dimensional size of the conducting body $(\mathrm{m})$ & $u, v$ & velocity components $\left(\mathrm{ms}^{-1}\right)$ \\
$D$ & non-dimensional size of the solid body & $U, V$ & non-dimensional velocity components \\
$g$ & acceleration due to gravity $\left(\mathrm{ms}^{-2}\right)$ & $V_{0}$ & lid velocity $\left(\mathrm{ms}^{-1}\right)$ \\
$G r$ & Grashof number & $\bar{V}$ & cavity volume $\left(\mathrm{m}^{3}\right)$ \\
$H a$ & Hartmann number & $x, y$ & Cartesian coordinates $(\mathrm{m})$ \\
$J$ & Joule heating parameter & $X, Y$ & non-dimensional Cartesian coordinates \\
$k$ & thermal conductivity of fluid $\left(\mathrm{Wm}^{-1} \mathrm{~K}^{-1}\right)$ & $G r e e k$ & symbols \\
$k_{s}$ & thermal conductivity of solid $\left(\mathrm{Wm}^{-1} \mathrm{~K}^{-1}\right)$ & $\alpha$ & thermal diffusivity $\left(\mathrm{m}^{2} \mathrm{~s}^{-1}\right)$ \\
$K$ & thermal conductivity ratio of solid and fluid & $\beta$ & thermal expansion coefficient $\left(\mathrm{K}^{-1}\right)$ \\
$L$ & length of the cavity $(\mathrm{m})$ & $\theta$ & non-dimensional temperature \\
$N u$ & Nusselt number & $\mu$ & dynamic viscosity of the fluid $\left(\mathrm{Kg} \mathrm{m}^{-1} \mathrm{~s}^{-1}\right)$ \\
$p$ & dimensional pressure $\left(\mathrm{Nm}{ }^{-2}\right)$ & $v$ & kinematic viscosity of the fluid $\left(\mathrm{m}^{2} \mathrm{~s}^{-1}\right)$ \\
$P$ & non-dimensional pressure & $\sigma$ & electrical conductivity of the fluid $\left(\Omega^{-1} \mathrm{~m}^{-1}\right)$ \\
$P r$ & Prandtl number & $\rho$ & density of the fluid $\left(\mathrm{Kg} \mathrm{m} \mathrm{m}^{-3}\right)$ \\
$R e$ & Reynolds number & $S u b s c r i p t s$ & \\
$R i$ & Richardson number & $a v$ & average \\
$T$ & dimensional temperature of fluid $(\mathrm{K})$ & $s$ & solid \\
$T_{\text {cold }}$ & dimensional temperature of lid $(\mathrm{K})$ & $c$ & centre \\
\hline
\end{tabular}




\section{Introduction}

Mixed convection flow and heat transfer in lid-driven cavities have been receiving an extensive attention in the literature. This attention stems from its importance in vast technological, engineering, and natural applications. Such applications include cooling of electronic devices, lubrication technologies, drying technologies, food processing, float glass production, flow and heat transfer in solar ponds, thermal-hydraulics of nuclear reactors and dynamics of lakes. Analysis of a mixed convection flow usually requires an understanding of the two limiting regimes. When current flows in a wire, the resistance of the wire causes a voltage drop along the wire. As a result electrical energy is lost. This lost electrical energy is converted into thermal energy called Joule heating. The heat transfer from a wavy surface is functional in several practical appliances for instance refrigeration system, radiator, louver, plurality of holes etc. A combined free and forced convection flow of an electrically conducting fluid in a cavity in presence of magnetic field and Joule heating effect is of special technical significance because of its frequent occurrence in many industrial applications such as geothermal reservoirs, thermal insulations and petroleum reservoirs. These types of problems also arise in electronic packages, micro electronic devices during their operations.

It should be pointed out that viscous flow in wavy channels was first analyzed by Bums and Parkes (1967), while Goldstein and Sparrow (1977) used the naphthalene technique to measure local and average heat transfer coefficients in a corrugated wall channel. Kumar (2000) conducted a study of flow and thermal field inside a vertical wavy enclosure filled with a porous media. The author illustrated that the surface temperature was very sensitive to the drifts in the surface undulations, phase of the wavy surface and the number of considered waves. Adjlout et al. (2002) studied laminar natural convection in an inclined cavity with a heated undulated wall, i.e., smooth wave-like pattern. Their results concluded that the hot wall undulation affected the flow and heat transfer rate in the enclosure in which the local Nusselt number distribution resulted in a decrease of heat transfer rate as compared with the square enclosure. Furthermore, Wang and Chen (2002) analyzed forced convection in a wavy-wall channel and demonstrated the effects of wavy geometry, Reynolds number and Prandtl number on the skin friction and Nusselt number. Results were shown that the amplitudes of skin friction coefficient and Nusselt number had increased with an increase in the amplitude to wavelength ratio. The problem of unsteady laminar combined forced and free convection flow and heat transfer of an electrically conducting and heat generating or absorbing fluid in a vertical lid-driven cavity in the presence of a magnetic field was formulated by Chamkha (2002). Das and Mahmud (2003) conducted a numerical investigation of natural convection in an enclosure consisting of two isothermal horizontal wavy walls and two adiabatic vertical straight walls. Laminar flow through a two-dimensional square cavity with internal (volumetric) heat generation was analyzed numerically by Gustavo et al. (2003). Al-Amiri et al. (2007) investigated mixed convection heat transfer in lid-driven cavity with a sinusoidal wavy bottom surface. Their findings were that the corrugated liddriven cavity could be considered as an effective heat transfer mechanism at larger wavy surface amplitudes and low Richardson number.

Recently, mixed convection in a vented square cavity with a heat conducting horizontal solid circular cylinder was performed by Rahman et al. (2008), where the developed mathematical model was solved by employing Galerkin weighted residual method of finite element formulation. The authors investigated the effects of cylinder size and Richardson number on fluid flow and heat transfer performances. Moreover, Rahman et al. (2009) studied numerically magnetohydrodynamics mixed convection in a rectangular cavity with a heat conducting horizontal circular cylinder with joule heating. They found strong dependency of flow and thermal fields on magnetic field. Very recently, Goutam Saha (2010) formulated finite element simulation of magnetoconvection inside a sinusoidal corrugated enclosure with discrete isoflux heating from below. The author showed the effect of discrete heat source sizes on heat transfer for different values of Grashof number and Hartmann number.

To the best of the author's understanding, little attention has been paid to the problem of mixed convection flow and heat transfer in a lid-driven cavity that is heated from a wavy vertical surface. The objective of the present study is to examine the momentum and energy transport processes in a lid-driven cavity with wavy surface together with a heat conducting body. The results are shown in terms of parametric presentation of streamlines and isotherms for various pertinent dimensionless parameters such as magnetic parameter $\mathrm{Ha}$, Richardson number $R i$ and Prandtl number $\operatorname{Pr}$ offered by the wavy surface. Finally, the implications of the above parameters are also depicted on the average Nusselt number, average fluid temperature and temperature of body centre.

\section{Problem Formulation}

The treated problem is a two-dimensional square cavity with a side length $L$. The physical system considered in the present study is displayed in Fig.1. The top and bottom walls are taken adiabatic and impermeable while the 
Rehena Nasrin / Journal of Naval Architecture and Marine Engineering 7(2010) 13-24

vertical walls are maintained at uniform but different temperatures such that the right wall is assigned to temperature $T_{\text {hot }}$ while the left wall is subjected to temperature $T_{\text {cold }}$. Under all circumstances $T_{\text {hot }}>T_{\text {cold }}$ condition is maintained. Furthermore, the left wall is assumed to slide from bottom to top at a constant speed $V_{0}$ and right wall is sinusoidal wavy pattern. A magnetic field of strength $B_{0}$ is acting in a transverse direction normal to the side walls. A heat conducting square solid body is centered of the cavity. As well, Joule heating effect is active here.

\section{Mathematical Formulation}

The functioning fluid is assumed to be Newtonian and incompressible with the flow is set to operate in the laminar mixed convection regime. The leading equations under Boussinesq approximation in dimensionless type are as follows:

$$
\begin{aligned}
& \frac{\partial U}{\partial X}+\frac{\partial V}{\partial Y}=0 \\
& U \frac{\partial U}{\partial X}+V \frac{\partial U}{\partial Y}=-\frac{\partial P}{\partial X}+\frac{1}{R e}\left(\frac{\partial^{2} U}{\partial X^{2}}+\frac{\partial^{2} U}{\partial Y^{2}}\right) \\
& U \frac{\partial V}{\partial X}+V \frac{\partial V}{\partial Y}=-\frac{\partial P}{\partial Y}+\frac{1}{R e}\left(\frac{\partial^{2} V}{\partial X^{2}}+\frac{\partial^{2} V}{\partial Y^{2}}\right)+R i \theta-\frac{H a^{2}}{R e} V \\
& U \frac{\partial \theta}{\partial X}+V \frac{\partial \theta}{\partial Y}=\frac{1}{\operatorname{RePr}}\left(\frac{\partial^{2} \theta}{\partial X^{2}}+\frac{\partial^{2} \theta}{\partial Y^{2}}\right)+J V^{2} \\
& \frac{K}{\operatorname{RePr}}\left(\frac{\partial^{2} \theta_{s}}{\partial X^{2}}+\frac{\partial^{2} \theta_{s}}{\partial Y^{2}}\right)=0
\end{aligned}
$$

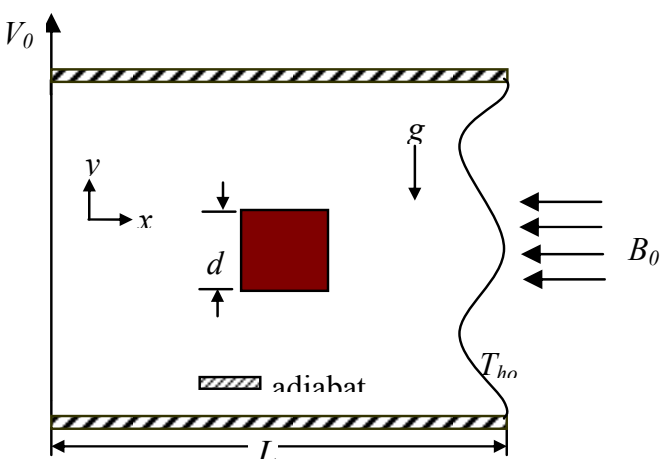

Fig. 1: Schematic diagram of the cavity

where $R e=\frac{V_{0} L}{v}, \operatorname{Pr}=\frac{v}{\alpha}, J=\frac{\sigma B_{0}^{2} \alpha}{\rho C_{p}\left(T_{\text {hot }}-T_{\text {cold }}\right)}$ and $R i=\frac{g \beta \Delta T L}{V_{0}^{2}}$ are Reynolds number, Prandtl number, Joule heating parameter and Richardson number respectively. $H a$ is Hartmann number which is defined as $H a^{2}=\frac{\sigma B_{0}^{2} L^{2}}{\mu}$. In addition, $K$ is the ratio of the thermal conductivity $\left(k_{s} / k\right)$

The nature of the vertical corrugated surface profile is assumed to mimic the following pattern $X-1=-A[1-\cos (2 \lambda \pi Y)]$ where $A$ is the dimensionless amplitude of the wavy surface and $\lambda$ is the number of undulations.

The above equations are non-dimensionalized upon incorporating the dimensionless variables given below:

$$
X=\frac{x}{L}, Y=\frac{y}{L}, U=\frac{u}{V_{0}}, V=\frac{v}{V_{0}}, P=\frac{p}{\rho V_{0}{ }^{2}}, D=\frac{d}{L}, \theta=\frac{\left(T-T_{\text {cold }}\right)}{\left(T_{\text {hot }}-T_{\text {cold }}\right)}, \theta_{s}=\frac{\left(T_{s}-T_{\text {cold }}\right)}{\left(T_{\text {hot }}-T_{\text {cold }}\right)}
$$

where the dimensionless quantities $X$ and $Y$ are the coordinates varying along horizontal and vertical directions respectively, $U$ and $V$ are the velocity components along the $X$ and $Y$ axes respectively, $\theta$ and $\theta_{s}$ are the temperature of fluid and solid respectively and $P$ is the pressure.

The boundary conditions for the present problem are specified as follows:

at the sliding lid: $U=0, V=1, \theta=0$; at all horizontal walls: $U=0, V=0, \frac{\partial \theta}{\partial N}=0$

at the right vertical wavy wall: $U=V=0, \theta=1$; At the cylinder boundary: $U=0, V=0$

At the fluid-solid interface: $\left(\frac{\partial \theta}{\partial N}\right)_{\text {fluid }}=K\left(\frac{\partial \theta_{s}}{\partial N}\right)_{\text {solid }}$ 
Rehena Nasrin / Journal of Naval Architecture and Marine Engineering 7(2010) 13-24

The rate of heat transfer is computed at the wavy wall and is expressed in terms of the local Nusselt number $\overline{N u}$ as $\overline{N u}=\frac{h L}{k}=-\frac{\partial \theta}{\partial N} L$

where, $N$ represents the coordinate direction normal to the surface. The dimensionless normal temperature gradient can be written as

$\frac{\partial \theta}{\partial N}=\frac{1}{L} \sqrt{\left(\frac{\partial \theta}{\partial X}\right)^{2}+\left(\frac{\partial \theta}{\partial Y}\right)^{2}}$

while the average Nusselt number $(\mathrm{Nu})$ is obtained by integrating the local Nusselt number along the vertical wavy surface and is defined by

$N u=\frac{1}{S} \int_{0}^{S} \overline{N u} d N$

where $S$ is the total chord length of the wavy surface.

Average temperature of the fluid in the enclosure is defined by $\theta_{a v}=\int \theta d \bar{V} / \bar{V}$, where $\bar{V}$ is the cavity volume.

\section{Computational Procedure}

By using the Galerkin weighted residual finite element technique, the momentum and energy balance equations have been solved that are the combinations of mixed elliptic-parabolic system of partial differential equations. Due to mass conservation, the continuity equation has been used as a constraint. The basic unknowns for the above differential equations are the velocity components $U, V$, the temperature $\theta$ and the pressure $P$. For the development of the finite element equations, the six nodes triangular element is used in this work. All six nodes are associated with velocities as well as temperature. Only the three corner nodes are associated with pressure. This means that a lower order polynomial is chosen for pressure and which is satisfied through continuity equation. The velocity components and the temperature profile and linear interpolation for the pressure distribution according to their highest derivative orders for the differential equations (2) - (5) are as

$U(X, Y)=N_{\beta} U_{\beta}, V(X, Y)=N_{\beta} V_{\beta}, \theta(X, Y)=N_{\beta} \theta_{\beta}, \theta_{s}(X, Y)=N_{\beta} \theta_{s_{\beta}}, P(X, Y)=H_{\lambda} P_{\lambda}$,

where $\beta=1,2, \ldots \ldots, 6$ and $\lambda=1,2,3$.

Substituting the element velocity components, the temperature and the pressure distributions in the Equations (2) - (5), the finite element equations can be written in the form

$$
\begin{aligned}
& K_{\alpha \beta \gamma}{ }^{x} U_{\beta} U_{\gamma}+K_{\alpha \beta \gamma} y V_{\gamma} U_{\gamma}+M_{\alpha \mu}{ }^{x} P_{\mu}+\frac{1}{\operatorname{Re}}\left(S_{\alpha \beta} x x+S_{\alpha \beta} y y\right) U_{\beta}=0 \\
& K_{\alpha \beta \gamma}{ }^{x} U_{\beta} V_{\gamma}+K_{\alpha \beta \gamma} V_{\beta} V_{\gamma}+M_{\alpha \mu}{ }^{y} P_{\mu}+\frac{1}{R e}\left(S_{\alpha \beta} x x+S_{\alpha \beta}^{y y}\right) V_{\beta} \\
& -\operatorname{Ri} K_{\alpha \beta} \theta_{\beta}+\frac{H a^{2}}{\operatorname{Re}} K_{\alpha \beta} V_{\beta}=0 \\
& K_{\alpha \beta \gamma}{ }_{\alpha \beta} U_{\beta} \theta_{\gamma}+K_{\alpha \beta \gamma} V_{\beta} \theta_{\gamma}+\frac{1}{\operatorname{RePr}}\left(S_{\alpha \beta}{ }^{x x}+S_{\alpha \beta} y y\right) \theta_{\beta}-J K_{\alpha \beta} V_{\beta}^{2}=0 \\
& \frac{K}{\operatorname{RePr}}\left(S_{\alpha \beta^{x x}}+S_{\alpha \beta} y y\right) \theta_{s \beta}=0
\end{aligned}
$$

where the coefficients in element matrices are in the following form of the integrals over the element area

$$
\begin{aligned}
& K_{\alpha \beta^{x}}=\int_{A} N_{\alpha} N_{\beta, x} d A, \quad K_{\alpha \beta^{y}}=\int_{A} N_{\alpha} N_{\beta, y} d A, \quad K_{\alpha \beta \gamma}=\int_{A} N_{\alpha} N_{\beta} N_{\gamma, x} d A, \quad K_{\alpha \beta \gamma^{y}}=\int_{A} N_{\alpha} N_{\beta} N_{\gamma, y} d A, \\
& K_{\alpha \beta}=\int_{A} N_{\alpha} N_{\beta} d A, \quad S_{\alpha \beta^{x x}}=\int_{A} N_{\alpha, x} N_{\beta, x} d A, \quad S_{\alpha \beta}^{y y}=\int_{A} N_{\alpha, y} N_{\beta, y} d A, \quad M_{\alpha \mu^{x}}=\int_{A} H_{\alpha} H_{\mu, x} d A, \\
& M_{\alpha \mu^{y}}=\int_{A} H_{\alpha} H_{\mu, y} d A
\end{aligned}
$$


Rehena Nasrin / Journal of Naval Architecture and Marine Engineering 7(2010) 13-24

Using Newton-Raphson method of Reddy (1993), the set of non-linear algebraic equations (6) - (9) are transferred into linear algebraic equations. Finally, these linear equations are solved by applying Triangular Factorization method and reduced integration technique of Zeinkiewicz et al. (1971). The application of this simulation is well described by Taylor and Hood (1973) and Dechaumphai (1999).

\section{Grid Sensitivity Test}

A grid independence test is reported with $H a=15, R e=100, J=1, K=7, A=0.05, D=0.2, \lambda=2, R i=0.1$ and $\operatorname{Pr}=0.73$ in order to decide the suitable grid size for this study. The following five kinds of meshes are considered for the grid sensitivity investigation. These grid densities are 3918 nodes, 436 elements; 9373 nodes, 1064 elements; 15465 nodes, 1768 elements; 31514 nodes, 3636 elements and 49592 nodes, 5748 elements. The extreme values of the average Nusselt number $(\mathrm{Nu})$ that relates to the heat transfer rate of the heated surface and average temperature $\left(\theta_{a v}\right)$ of the fluid in the enclosure are used as sensitivity measures of the correctness of the solution. They are selected as the supervising variables for the grid liberty test. The addiction of the quantities $N u$ and $\theta_{a v}$ on the grid size and the computational time are shown in Table 1. The current formulation is performed with 31514 nodes and 3636 elements grid system by considering both the accuracy of numerical values and computational time.

Table 1: Grid Sensitivity Check at $H a=15, \operatorname{Pr}=0.73, R i=0.1, \operatorname{Re}=100, A=0.05, K=7, D=0.2, \lambda=2$ and $J=1$

\begin{tabular}{|c|c|c|c|c|c|}
\hline $\begin{array}{c}\text { Nodes } \\
\text { (elements) }\end{array}$ & $\begin{array}{c}3918 \\
(436)\end{array}$ & $\begin{array}{c}9373 \\
(1064)\end{array}$ & $\begin{array}{c}15465 \\
(1768)\end{array}$ & $\begin{array}{c}31514 \\
(3636)\end{array}$ & $\begin{array}{c}49592 \\
(5748)\end{array}$ \\
\hline$N u$ & 1.597893 & 1.602778 & 1.618247 & 1.622647 & 1.622647 \\
\hline$\theta_{a v}$ & 0.336241 & 0.325121 & 0.310542 & 0.306112 & 0.306112 \\
\hline Time (s) & 246.269 & 308.603 & 398.167 & 445.321 & 550.379 \\
\hline
\end{tabular}

\section{Results and Discussion}

The characteristics of the flow and temperature fields in the lid-driven cavity are examined by exploring the effects of magnetic parameter, Richardson number and Prandtl number. Such field variables are analyzed by outlaying the steady state version of the streamlines, temperature distribution, average temperature of fluid and temperature of body centre as well as the average Nusselt number. In the present numerical study, the following parametric domains of the dimensionless group is considered: $0 \leq H a \leq 55,0.73 \leq \operatorname{Pr} \leq 7,0.1 \leq R i \leq 10, A=$ $0.05, \operatorname{Pr}=0.73, \lambda=2, \operatorname{Re}=100, D=0.2, K=7$ and $J=1$.

For the variations of $\mathrm{Ha}$ and $\mathrm{Ri}$, the overall features of the streamlines are depicted in Fig. 2, where $\mathrm{Pr}=0.73$, $R e=100, K=7$ and $J=1$ are kept fixed. In the absence of the magnetic field $(H a=0)$ and the natural convection effect $(R i=0.1)$, the fluid flow is characterized by a primary rotating uni-cellular vortex occupying the bulk of the cavity generated by the movement of the left lid. The size of the vortex remains unaffected, but the core of the vortex shifted towards the left top corner of the cavity with increasing $H a$ up to 30 and the core divided into two parts positioned at the bottom and top corner near the left wall at the highest value of $H a$. When natural convection and forced convection become equally dominant, namely $R i=1$, the velocity field exists a primary recirculating clockwise rotating vortex. In addition, two minor vortices tend to form near the corrugated top and bottom corners by the buoyant force at $\mathrm{Ha}=55$. As $\mathrm{Ha}$ decreases from 55 to 30 , the minor counter clockwise vortices merge into a two cellular single one and become comparatively large. As a result the clockwise rotating eddy turns into smaller in size. Furthermore, the size of the clockwise vortex decreases and counter clockwise eddy increases with falling $H a$ for $R i=1$. This is because application of a transverse magnetic field has the tendency to slow down the movement of the buoyancy-induced flow in the cavity. When $R i=10$, the role of natural convection is far more compared to the forced convection effect, consequently the size of the counter clockwise eddy is larger than that of earlier case for each $\mathrm{Ha}$.

The corresponding effects on the temperature field are shown in Fig. 3. The isotherms are almost parallel to the vertical walls for the highest value of $H a$ at all $R i$, indicating that most of the heat transfer process is carried out by conduction. However, some deviations in the conduction dominated isothermal lines are initiated near the left top surface of the cavity for $H a=30$. This distortion increases quickly with decreasing $H a$ at $R i=0.1$. The isothermal lines are dominated by conduction and mixed convection heat transfer is observed in the cavity for 
Rehena Nasrin / Journal of Naval Architecture and Marine Engineering 7(2010) 13-24 different $H a$ at $R i=1$. But when $R i=10$, the temperature field is drastically changed with diminishing $H a$. Moreover, the formation of the thermal boundary layer near the left cold wall is to be initiated for the lower values of $H a$. This is owing to the dominating influence of the convective current in the cavity.
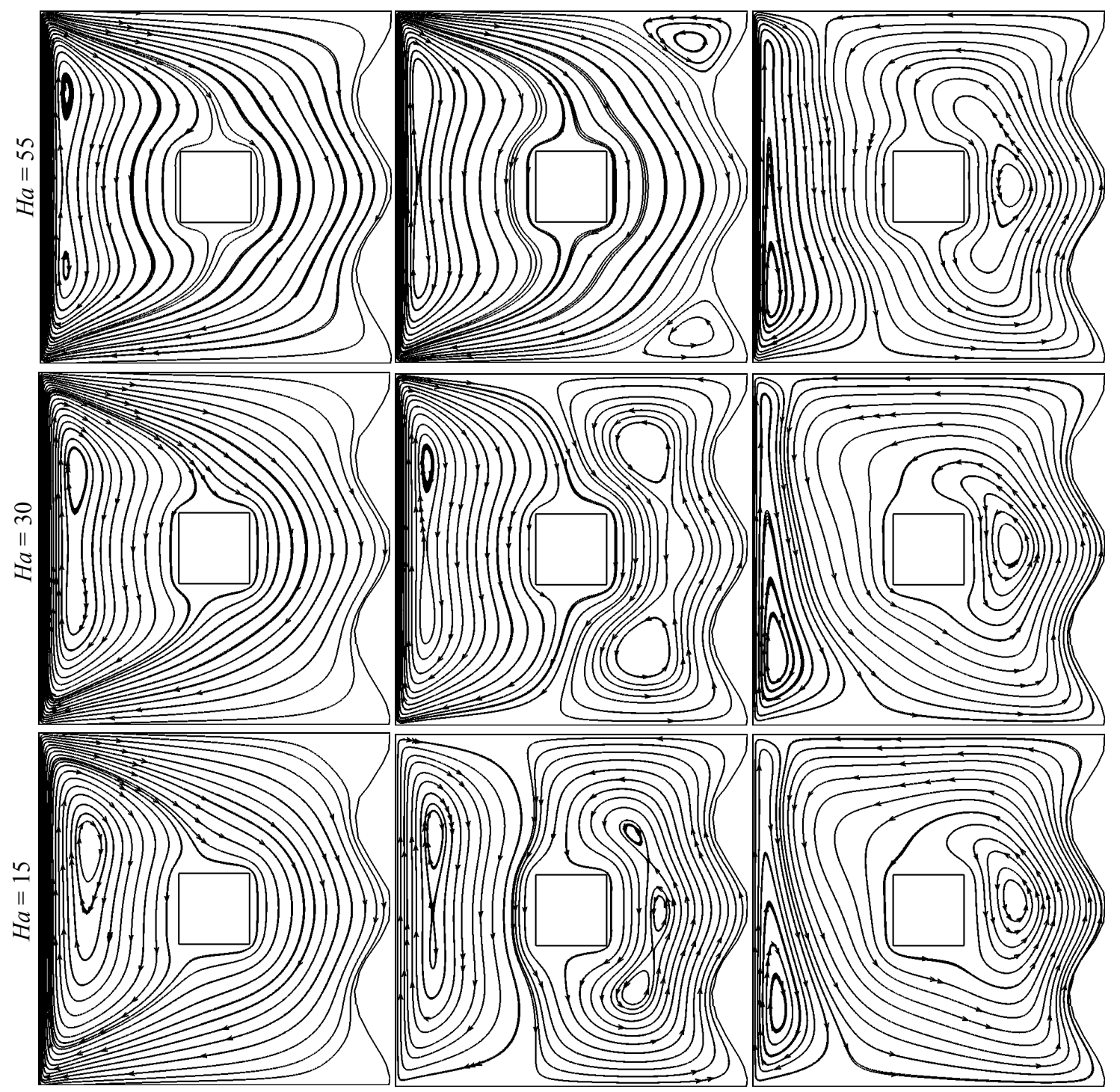

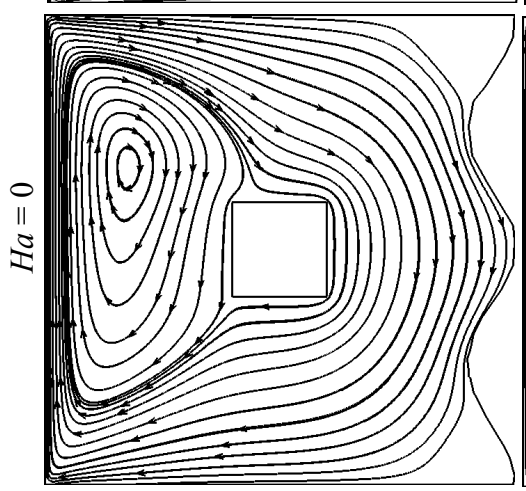

$R i=0.1$

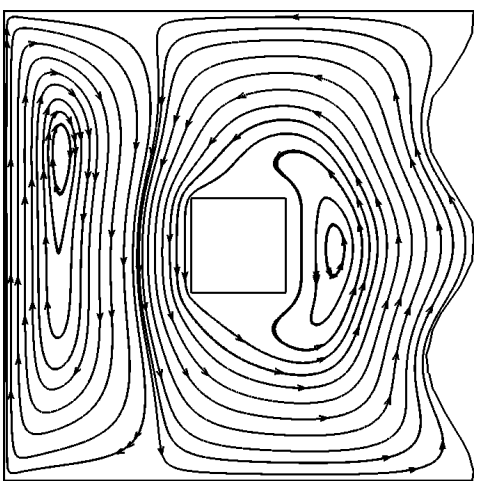

$R i=1$

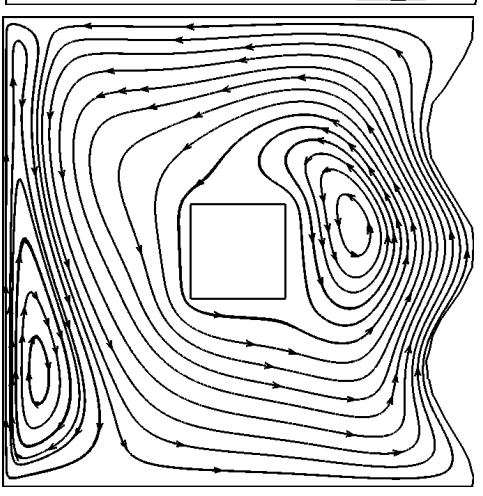

$R i=10$

Fig. 2: Streamlines for different values of $H a$ and $R i$, while $R e=100, P r=0.73, K=7$ and $J=1$ 
Rehena Nasrin / Journal of Naval Architecture and Marine Engineering 7(2010) 13-24
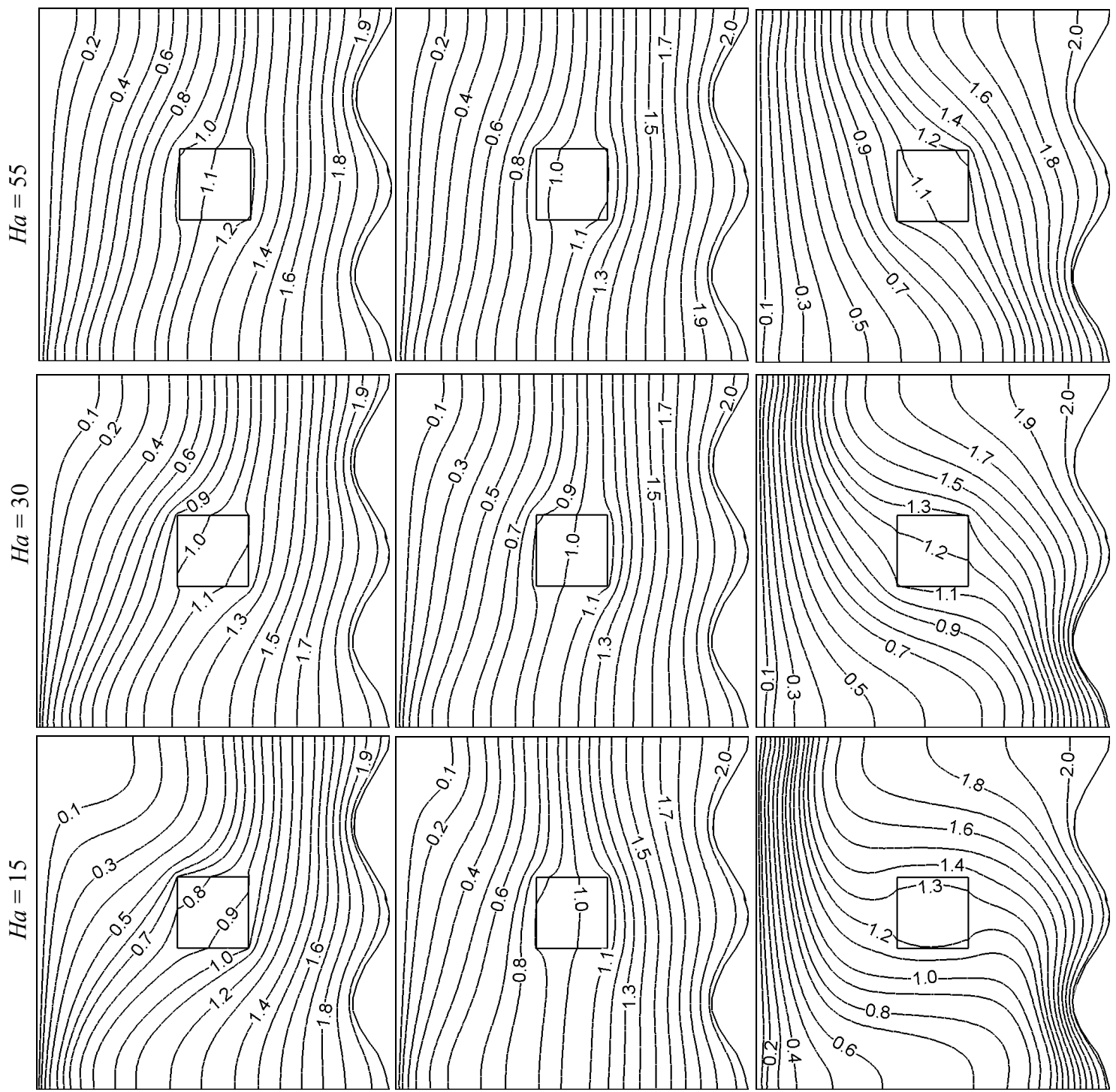

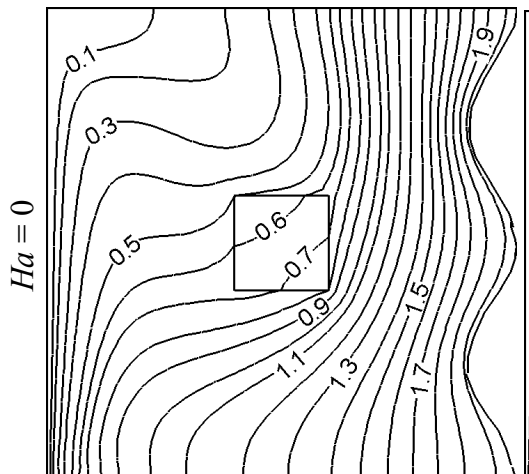

$R i=0.1$

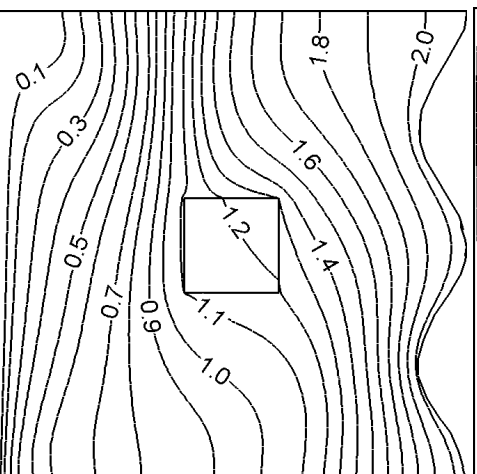

$R i=1$

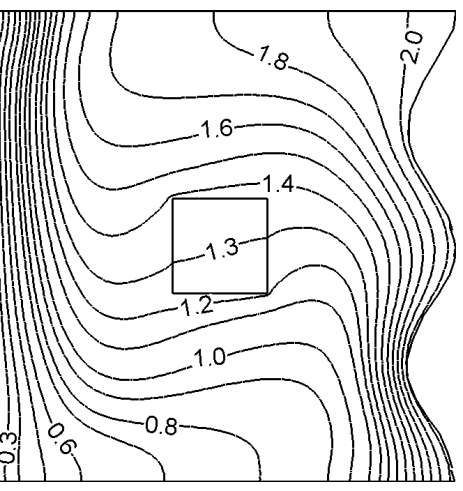

Fig. 3: Isotherms for various $H a$ and $R i$, while $R e=100, P r=0.73, K=7$ and $J=1$

Fig. 4 exposes the influence of Hartmann number on average Nusselt number $(\mathrm{Nu})$ at the hot wavy wall, average temperature $\left(\theta_{a v}\right)$ of the fluid in the cavity and the temperature $\left(\theta_{c}\right)$ at the body center along with Richardson 
Rehena Nasrin / Journal of Naval Architecture and Marine Engineering 7(2010) 13-24

number. $N u$ goes down very rapidly up to a certain value of $R i$ and grows up gradually with increasing $R i$ for the lower values of $\mathrm{Ha}(0,15$ and 55). But it decreases mildly at $\mathrm{Ha}=30$. Also, $\mathrm{Nu}$ is the uppermost in the pure forced convection area at $H a=0$ and in the natural convection dominated region at $H a=30$. On the other hand, $\theta_{a v}$ and $\theta_{c}$ increase sharply with the variation of $R i$ for the lower $H a(0,15$ and 30) and remain flatten at the highest $\mathrm{Ha}$.
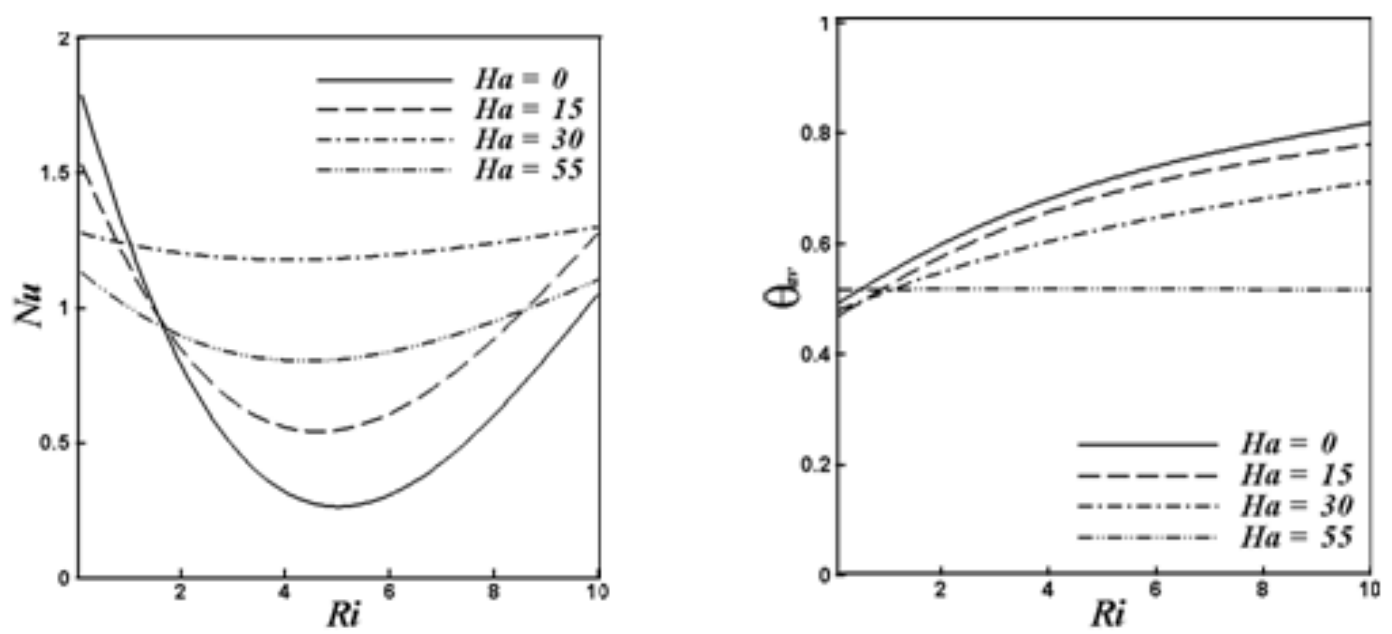

Fig. 4.a: Effect of $\mathrm{Ha}$ on $\mathrm{Nu}$ and $\theta_{a v}$ for various $R i$, while $R e=100, \operatorname{Pr}=0.73, K=7$ and $J=1$

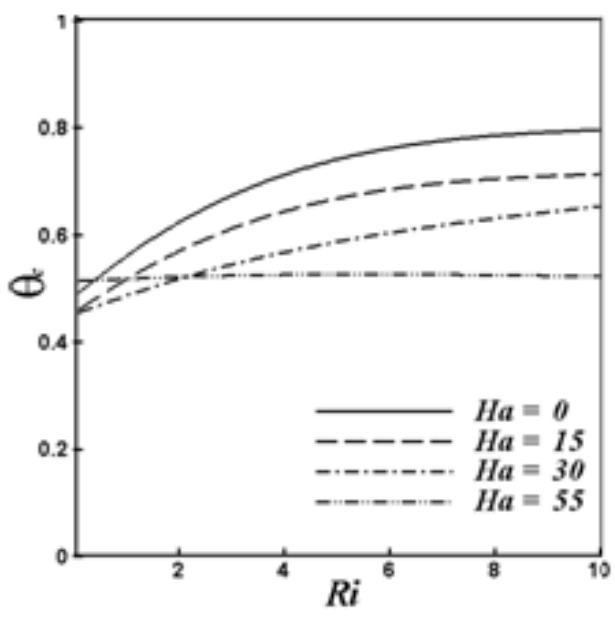

Fig. 4.b: Effect of $\mathrm{Ha}$ on $\theta_{c}$ for various $\mathrm{Ri}$, while $\operatorname{Re}=100, \operatorname{Pr}=0.73, K=7$ and $J=1$
The influence of Prandtl number on the flow field at three different values of $R i$ is shown in Fig. 5, while $R e=100$, $H a=15, J=1$ and $K=7$. The streamlines for all values of $\operatorname{Pr}(\operatorname{Pr}=0.73,1.73,3.5$ and 7$)$ and low $R i(R i=0.1)$ are found to be established due to the shear induced force by the moving lid only. Next at $R i=1$, the balance between the shear and buoyancy effect is manifested in the formation of two vortices inside the cavity. It is also seen that the shear effect produces the clockwise vortex, which is comparatively small than that of the three-cellular counter clockwise eddy produced by the buoyant force. As the Richardson number rises further to 10 , the heat transfer is mostly by convection in the cavity, as a result the threecellular counter clockwise eddy becomes uni-cellular and large enough and the clockwise vortex becomes shrink in size at each $\operatorname{Pr}$ considered. It becomes visible from Fig. 5 that varying $\operatorname{Pr}$ does not perturb the global flow at each $R i$.

Fig. 6 provides the corresponding effects on temperature field. The isotherms at very low $P r$ and lower $R i(R i=0.1$, 1) become almost parallel to the vertical walls, resembling the conduction like heat transfer in the cavity. On further

increasing $R i$ up to 10 , the degree of distortion from the conduction heat transfer is very noticeable and the bend in isothermal lines appears due to the high convective current inside the cavity. The isotherms depart from the hot corrugated sinusoidal wall and begin to crowd near the left lid forming a thin thermal boundary layer in the cavity with all $R i$ and the higher Prandtl number $(P r=1.73,3.5$ and 7).

The deviations of average Nusselt number $(N u)$ at the heated wall, average temperature $\left(\theta_{a v}\right)$ of the fluid in the enclosure and temperature $\left(\theta_{c}\right)$ at the body center for $\operatorname{Pr}$ and $R i$ are depicted in Fig. 7. For all values of $\operatorname{Pr}$ the average Nusselt number devalues rapidly up to $R i=4$ and increases slowly with $R i$ in the free convection dominated area. In addition, maximum value of $N u$ is found for the highest $\operatorname{Pr}$ at all $R i$. On the other hand, $\theta_{a v}$ and $\theta_{c}$ rise smoothly for each $\operatorname{Pr}$ with mounting $R i$. Minimum values of $\theta_{a v}$ and $\theta_{c}$ are observed at the highest $P r$ in the forced convection dominated region and at the lowest $\operatorname{Pr}$ in the free convection dominated regime. 
Rehena Nasrin / Journal of Naval Architecture and Marine Engineering 7(2010) 13-24
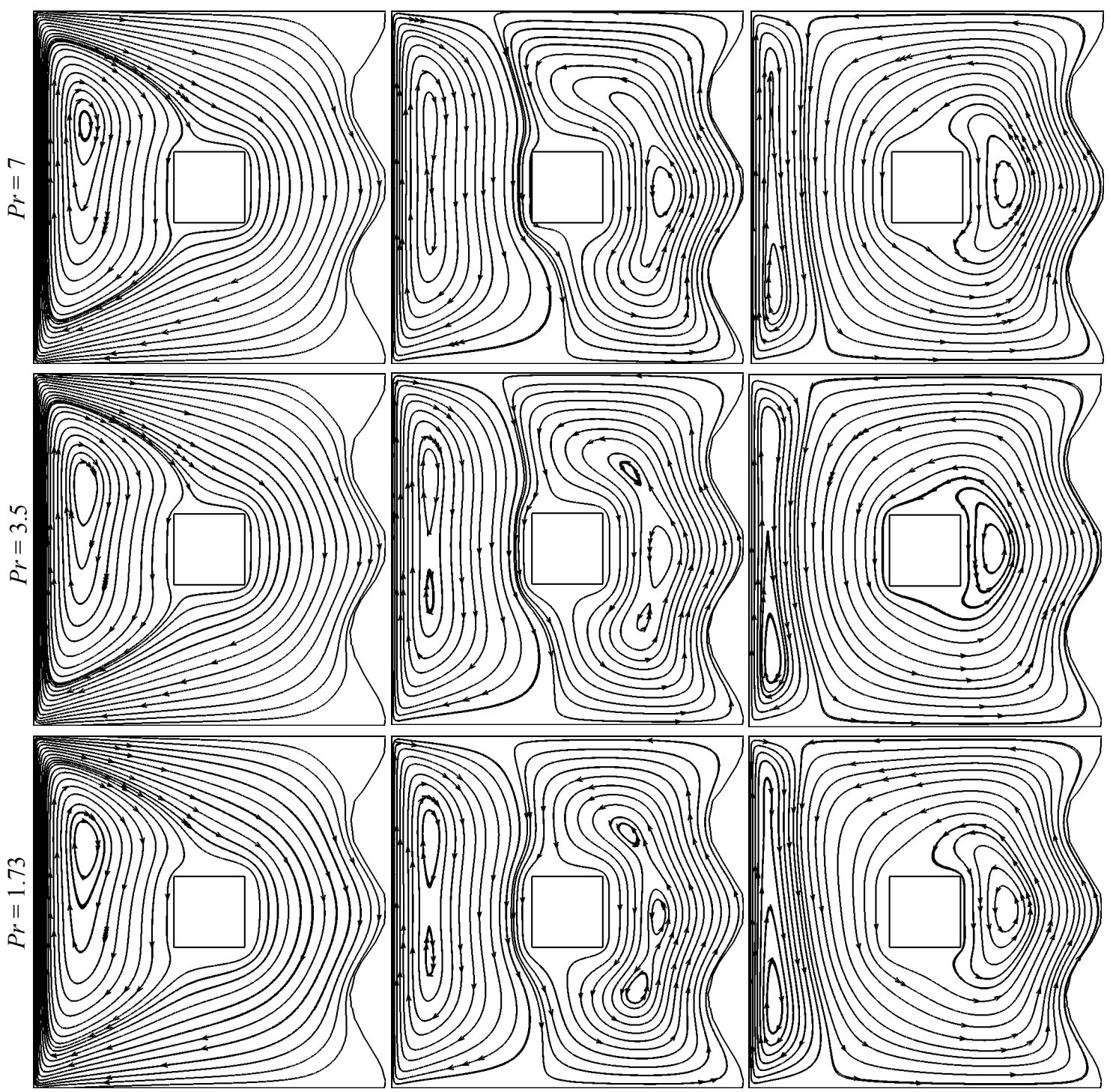

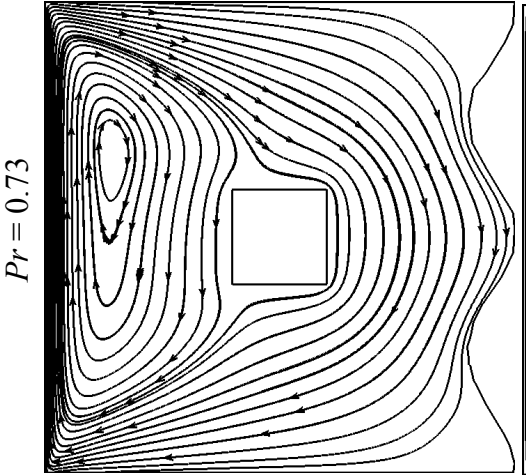

$R i=0.1$

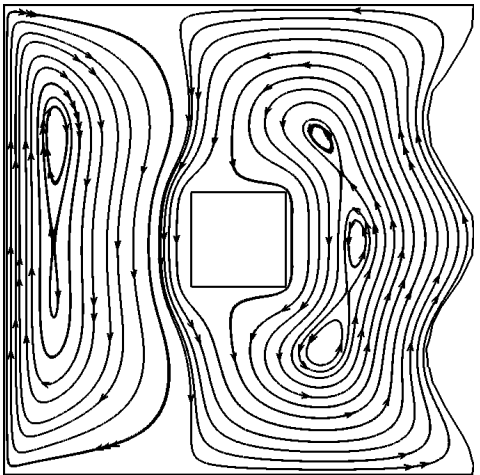

$R i=1$

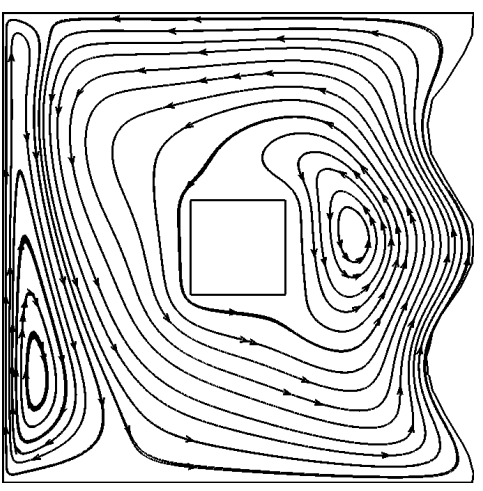

Fig. 5: Streamlines for different values of $\operatorname{Pr}$ and $R i$, while $R e=100, H a=15, K=7$ and $J=1$ 
Rehena Nasrin / Journal of Naval Architecture and Marine Engineering 7(2010) 13-24
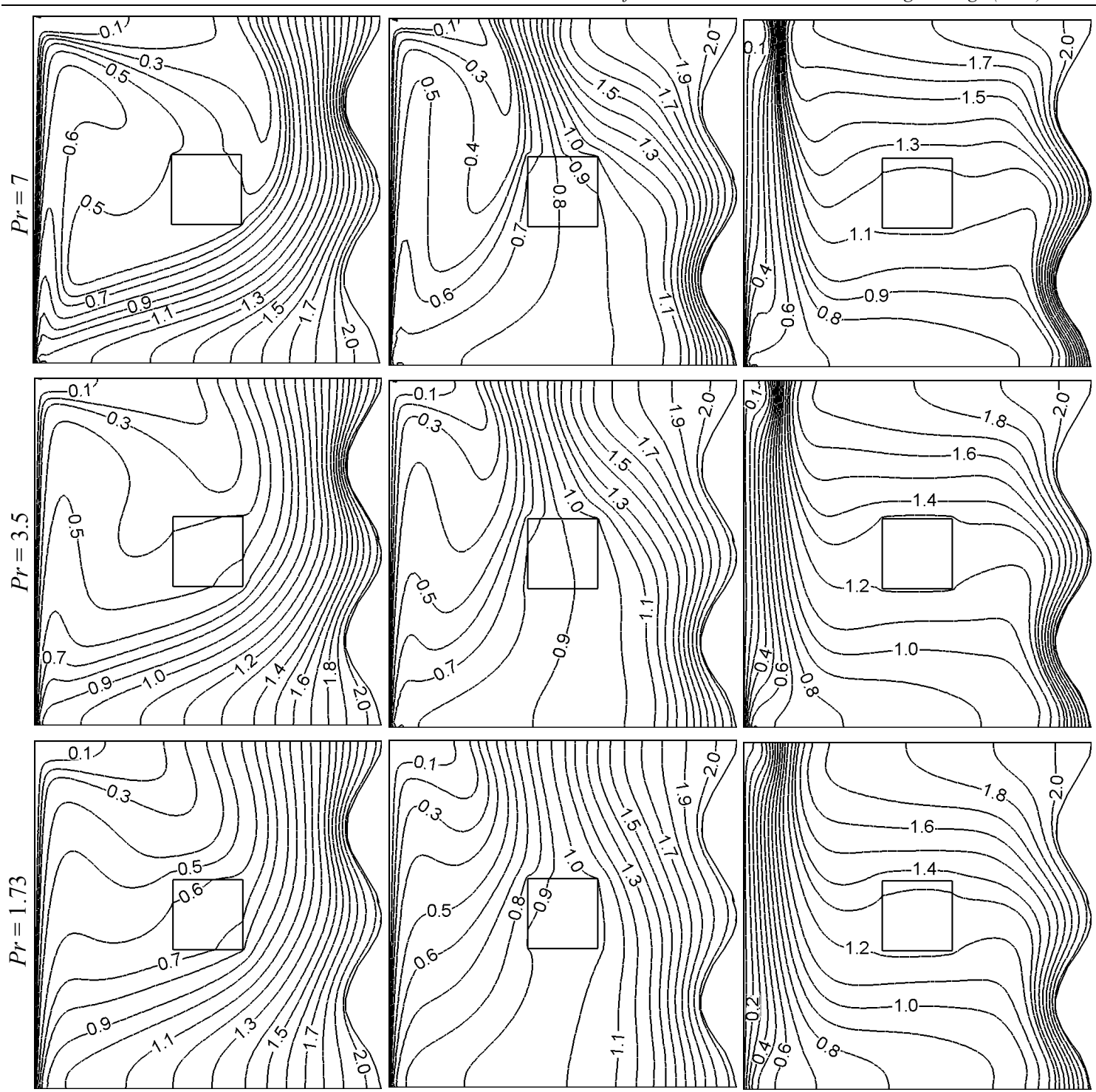

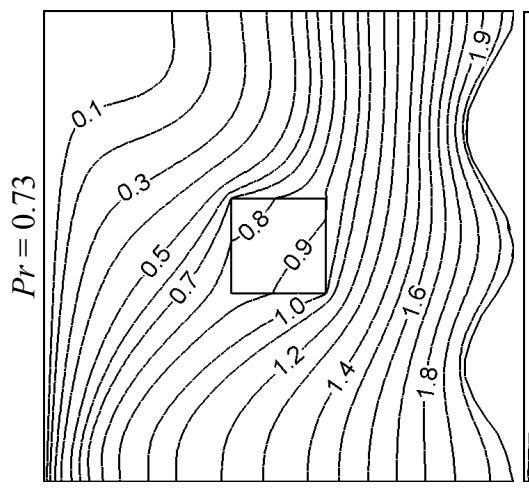

$R i=0.1$

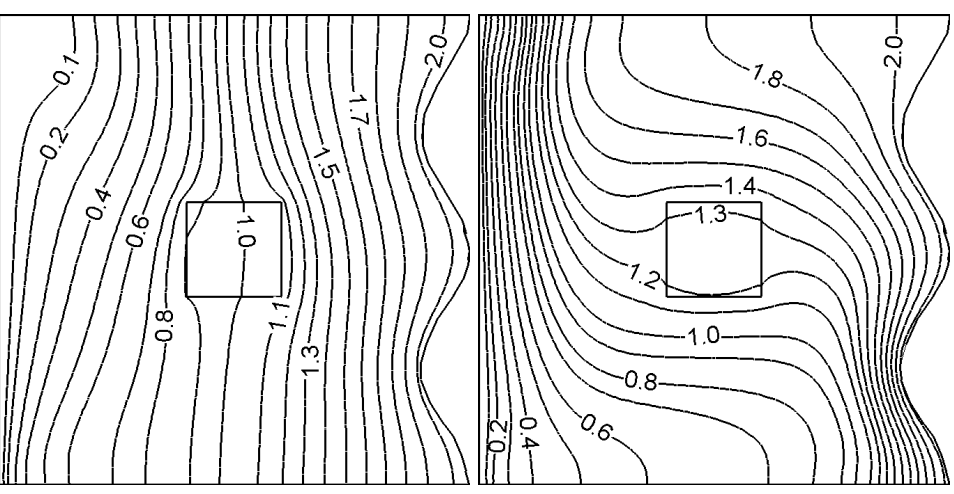

$R i=1$
$R i=10$

Fig. 6: Isotherms for different values of $\operatorname{Pr}$ and $R i$, while $R e=100, H a=15, K=7$ and $J=1$ 

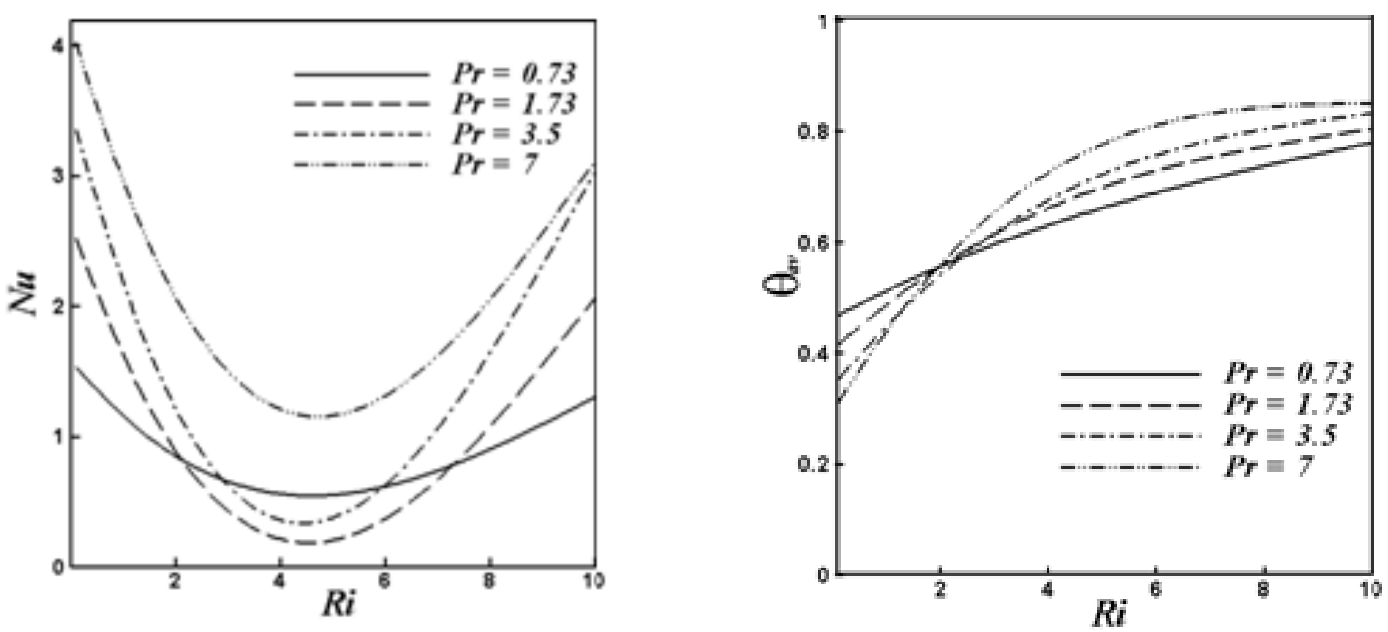

Fig. 7.a: Effect of $\operatorname{Pr}$ on $N u$, and $\theta_{a v}$ for various $R i$, while $R e=100, H a=15, K=7$ and $J=1$

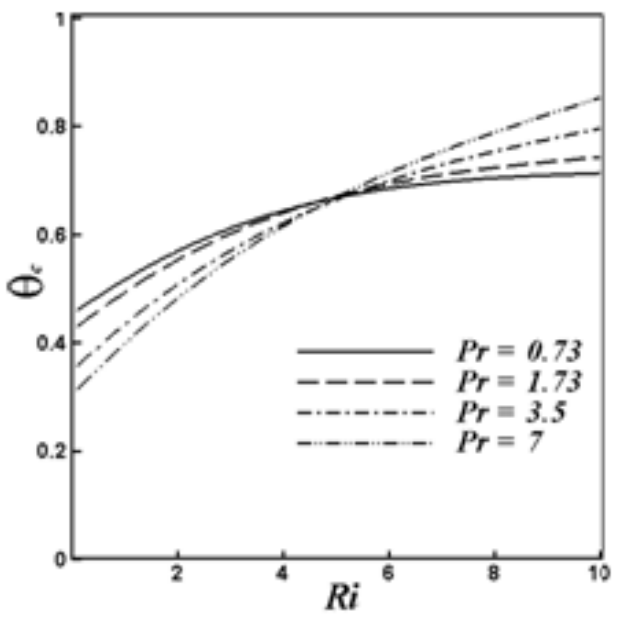

Fig. 7.b: Effect of $\operatorname{Pr}$ on $\theta_{c}$ for various $R i$, while $R e=100, H a=15, K$ $=7$ and $J=1$

\section{Conclusion}

This work is focused on the study of mixed magnetoconvection of fluid enclosed in a lid-driven cavity heated from wavy vertical surface. Effects of magnetic parameter and Prandtl number at the three convection regimes are highlighted to explore their impacts on flow structure and heat transfer characteristics.

- The influence of $\mathrm{Ha}$ does not affect significantly the thermal current activities. But the flow pattern changes dramatically owing to the hindrance of the imposed magnetic field.

- The overall flow behavior is not disturbed and isothermal lines become more bend due to the variation of $\operatorname{Pr}$.

- Increasing $R i$ leads to grow more eddy in velocity field and to develop thermal boundary layer near left lid in the cavity.

- $\quad N u$ is discovered to be most and least in the absence of magnetic field for $R i=0.1$ and 10 respectively, but it is always superior for the largest $\operatorname{Pr}$. Lesser $\theta_{a v}$ and $\theta_{c}$ are seen for lower values of $\mathrm{Ha}$ (15 and 30) and $P r=7$ in the forced convection dominated area and for $H a=55$ and $P r=0.73$ in the free convection dominated region.

\section{References}

Adjlout, L., Imine, O., Azzi, A., Belkadi, M. (2002): Laminar natural convection in an inclined cavity with a wavy-wall, International Journal of Heat and Mass Transfer, Vol. 45, No. 10, pp. 2141-2152.

Al-Amiri, A., Khanafer, K., Bull, J., Pop, Ioan (2007): Effect of sinusoidal wavy bottom surface on mixed convection heat transfer in a lid-driven cavity, International Journal of Heat and Mass Transfer, Vol. 50, No. (910), pp. 1771-1780. doi:10.1016/j.ijheatmasstransfer.2006.10.008

Bums, J.C., Parkes, T. (1967): Peristaltic motion, Journal of Fluid Mechanics, Vol. 29, pp. 731-743. doi: $\underline{10.1017 / \mathrm{S} 0022112067001156}$

Buscaglia, Gustavo C. and Dari, Enzo A. (2003): Numerical investigation of flow through a cavity with internal heat generation, Numerical Heat Transfer, Part A, Vol. 43, No. 5, pp. 525-541. doi: 10.1080/10407780307317

Chamkha, A.J. (2002): Hydromagnetic combined convection flow in a vertical lid-driven cavity with internal heat generation or absorption, Numerical Heat Transfer, Part A, Vol. 41, No. 5, pp. 529-546. 
Rehena Nasrin / Journal of Naval Architecture and Marine Engineering 7(2010) 13-24

Das, P.K., Mahmud, S. (2003): Numerical investigation of natural convection inside a wavy enclosure, International Journal of Thermal Science, Vol. 42, No. 4, pp. 397-406. doi: 10.1016/S1290-0729(02)00040-6

Dechaumphai, P. (1999): Finite Element Method in Engineering, 2nd ed., Chulalongkorn University Press, Bangkok.

Goldstein, J.L., Sparrow, E.M. (1977): Heat/mass transfer characteristics for flow in a corrugated wall channel, ASME Journal of Heat Transfer, Vol. 99, pp. 187-195.

Kumar, B.V.R. (2000): A study of free convection induced by a vertical wavy surface with heat flux in a porous enclosure, Numerical Heat Transfer, Part A, Vol. 37, No. 5, pp. 493-510.

Rahman, M.M., Alim, M.A., Chowdhury, M.K. (2009): Magnetohydrodynamics mixed convection around a heat conducting horizontal circular cylinder in a rectangular cavity with joule heating, Journal of Scientific Research, Vol. 1, No. 3, pp. 461-472. doi: 10.3329/jsr.v1i3.2597

Rahman, Md. M., Alim, M.A., Saha, Sumon and Chowdhury, M.K. (2008): Mixed convection in a vented square cavity with a heat conducting horizontal solid circular cylinder, Journal of Naval Architecture and Marine Engineering, Vol. 5, No. 2, pp. 37-46. doi: 10.3329/jname.v5i2.2504

Reddy, J.N. (1993): An Introduction to Finite Element Analysis, McGraw-Hill, New-York.

Saha, Goutam (2010): Finite element simulation of magnetoconvection inside a sinusoidal corrugated enclosure with discrete isoflux heating from below, International Communications in Heat and Mass Transfer, Vol. 37, No. 4, pp. 393-400. doi:10.1016/j.icheatmasstransfer.2009.12.001

Taylor, C., Hood, P. (1973): A numerical solution of the Navier-Stokes equations using finite element technique, Computers and Fluids, Vol. 1, No. 1, pp. 73-89.

Wang, C.-C., Chen, C.-K. (2002): Forced convection in a wavy-wall channel, International Journal of Heat and Mass Transfer, Vol. 45, No. 12, pp. 2587-2595. doi: 10.1016/S0017-9310(01)00335-0

Zeinkiewicz, O.C., Taylor, R.L., Too, J.M. (1971): Reduced integration technique in general analysis of plates and shells, International Journal for Numerical Methods in Engineering, Vol. 3, pp. 275-290. 